\title{
Lentinus edodes promotes fat removal in hypercholesterolemic mice
}

\author{
HYUN YANG, INHO HWANG, SUN KIM, EUI-JU HONG and EUI-BAE JEUNG \\ Laboratory of Veterinary Biochemistry and Molecular Biology, College of Veterinary Medicine, \\ Chungbuk National University, Cheongju, Chungbuk 361-763, Republic of Korea
}

Received April 16, 2013; Accepted October 3, 2013

DOI: 10.3892/etm.2013.1333

\begin{abstract}
Lentinus (L.) edodes (shiitake mushroom) is used as a traditional medicine in Asia. One of the components of L. edodes, eritadenine (an adenosine analog alkaloid), has been shown to reduce cholesterol levels. The hypocholesterolemic action of eritadenine appears to be achieved through the modification of hepatic phospholipid metabolism. In the present study, the effects of $L$. edodes in a mouse model of hypercholesterolemia were investigated. Hypercholesterolemia was induced by the consumption of a high-fat diet (HFD). The animals were divided into six groups, which were fed a normal diet, HFD alone, HFD containing eritadenine $[10 \mathrm{mg} / \mathrm{kg}$ of body weight (BW)] or HFD with 5,10 or $20 \%$ L. edodes, respectively, for 4 weeks (from 5 to 9 weeks of age). The mice in the six groups had similar BW gains. Total serum cholesterol (T-CHO), low-density lipoprotein (LDL) and triglyceride (TG) levels were increased in the HFD-fed group compared with those in the normal diet group. However, the levels of high-density lipoprotein (HDL) were not significantly altered. In mice treated with L.edodes $(5,10$ or $20 \%)$, the T-CHO, LDL and TG serum levels were reduced in a dose-dependent manner. The mRNA expression of cholesterol 7- $\alpha$-hydroxylase 1 (CYP7A1) was decreased in hypercholesterolemic mice and increased by eritadenine and L.edodes (5, 10 and 20\%) supplementation. In liver tissues, it was observed that lipid accumulation was reduced by treatment with eritadenine and L. edodes. In addition, it was revealed that the formation of atherosclerotic plaques due to the HFD was also suppressed by eritadenine and L.edodes. The results of the study indicated that the consumption of an HFD may inhibit CYP7A1 expression in the liver by increasing serum T-CHO, LDL and TG levels. L. edodes may help regulate lipid metabolism,
\end{abstract}

Correspondence to: Professor Eui-Bae Jeung, Laboratory of Veterinary Biochemistry and Molecular Biology, College of Veterinary Medicine, Chungbuk National University, Cheongju, Chungbuk 361-763, Republic of Korea

E-mail: ebjeung@chungbuk.ac.kr

Key words: Lentinus edodes, hypercholesterolemia, eritadenine, CYP7A1 suggesting that this fungus ameliorates hypercholesterolemia in mice by regulating CYP7A1 expression in the liver.

\section{Introduction}

Lentinus (L.) edodes (also know as shiitake) is a popular mushroom known to possess beneficial bioactivities. This mushroom is an important ingredient in Asian cuisine, due to its desirable taste and odor. In addition, the fungus has high levels of vitamins B and D (1). One of the bioactive compounds in L. edodes is eritadenine, which has been demonstrated to exert anti-hypercholemic effects in previous studies $(2,3)$. In addition, eritadenine may regulate lipid metabolism by inhibiting S-adenosyl homocysteine hydrolase activity (4-6).

The conversion of cholesterol into bile acids in the liver is important for eliminating cholesterol from the body. Bile acids are essential for the absorption and transport of lipid-soluble vitamins, steroids and xenobiotics (7-9). The main bile acid biosynthetic pathway is initiated by cholesterol 7- $\alpha$-hydroxylase 1 (CYP7A1), a cytochrome P450 heme enzyme (10). The transcription of CYP7A1 is inhibited by bile acid feedback and stimulated by dietary factors, such as cholesterol $(11,12)$. The expression of CYP7A1 is downregulated by sterol regulatory element-binding proteins (SREBPs) when plasma cholesterol levels are low and upregulated by the nuclear receptor liver $\mathrm{X}$ receptor (LXR) when cholesterol levels are high (13).

Dyslipidemia and hypercholesterolemia are among the numerous risk factors associated with diabetes and metabolic diseases, including fatty liver, obesity and atherosclerosis (14-16). Eritadenine (an adenosine analog alkaloid) and lentinacin (a purine alkaloid) have been shown to reduce cholesterol levels in rats by $25 \%$ following 7 days of oral administration at doses as low as $0.005 \%$ of the feed intake (17). The hypocholesterolemic action of eritadenine appears to be associated with the modification of hepatic phospholipid (PL) metabolism, through the induction of phosphatidylethanolamine N-methyltransferase deficiency (4). Dietary eritadenine also alters the fatty acid and molecular profiles of the liver and plasma by suppressing the conversion of linoleic acid into arachidonic acid and decreasing $\Delta 6$-desaturase activity (5). In the present study, the effects of $L$. edodes were investigated in a mouse model of hypercholesterolemia induced by the consumption of a high-fat diet (HFD) during the growth stage 
of the animals (between 4 and 6 weeks of age). The serum levels of total cholesterol (T-CHO) and triglyceride (TG) were measured. In addition, the expression of CYP7A1 in the liver was evaluated in order to study potential mechanisms underlying the hypocholesterolemic effects of L.edodes.

\section{Materials and methods}

Experimental animals. Imprinting Control Region (ICR) mice (5-week-old) were obtained from Koatech (Pyeongtaek, Republic of Korea). All animals were housed in polycarbonate cages and acclimated in an environmentally controlled room $\left(23 \pm 2^{\circ} \mathrm{C}, 50 \pm 10 \%\right.$ relative humidity, frequent ventilation and a 12-h light/dark cycle) prior to use. The mice $(n=60)$ were divided into six groups ( $\mathrm{n}=10$ per group). Hypercholesterolemia was induced in five of the groups by the consumption of an HFD (D12336; Research Diets, Inc., New Brunswick, NJ, USA) for 2 weeks (from 4 to 6 weeks of age).

To assess the ability of L.edodes to protect against hypercholesterolemia, the mice were fed the HFD alone as a negative control (NC), HFD with $10 \mathrm{mg} / \mathrm{kg}$ eritadenine (Santa Cruz Biotechnology, Inc., Santa Cruz, CA, USA) as a positive control (PC) and HFD supplemented with 5\% (LE5; w/w), 10\% (LE10; w/w) or $20 \%$ (LE20; w/w) L. edodes for 4 weeks (from 5 to 9 weeks of age). The remaining group (vehicle) was fed with D12337 pellets (Research Diets, Inc.) as a vehicle control. The compositions of the HFD (D12336) and control diet (D12337) are shown in Table I. Body weight (BW) was measured prior to and following the experimental feeding period. All animal experimental procedures were approved by the Ethics Committee of Chungbuk National University (Cheongju, Republic of Korea).

Serum and urine collection and biochemical analysis. Blood was collected from the abdominal aorta in each mouse, transferred to serum separator tubes (Microtainer tubes; Becton-Dickinson Co., San Jose, CA, USA) and centrifuged at $400 \mathrm{x}$ g for $15 \mathrm{~min}$. The serum was collected and stored in 200-ml aliquots. T-CHO, high-density lipoprotein (HDL), low-density lipoprotein (LDL) and TG levels in the serum were measured using a Hitachi 7080 auto-analyzer (Hitachi Science System Ltd., Ibaraki, Japan).

RNA extraction and quantitative polymerase chain reaction $(q P C R)$. Total RNA was extracted from the mouse liver using TRIzol ${ }^{\circledR}$ reagent (Invitrogen Life Technology, Carlsbad, CA, USA), in accordance with the manufacturer's instructions. RNA concentrations were measured with a microplate spectrophotometer (Epoch; BioTek Inc., Winooski, VT, USA) at $260 \mathrm{~nm}$. RNA quality was evaluated using electrophoresis in $1 \%$ agarose gels. Total RNA ( $1 \mathrm{mg}$ ) was reverse transcribed into first-strand complementary DNA (cDNA) using Moloney murine leukemia virus reverse transcriptase (Invitrogen Life Technology) and random primers (9-mer; Takara Bio, Otsu, Japan). Each cDNA sample $(1 \mathrm{ml})$ was amplified with $10 \mathrm{ml}$ 2X SYBR ${ }^{\circledR}$ Premix Ex Taq ${ }^{\mathrm{TM}}$ (Takara Bio) and 10 pmol of each primer. Amplification was performed in a 7300 Real-Time PCR System (Applied Biosystems, Foster City, CA, USA) with the following parameters: denaturation at $95^{\circ} \mathrm{C}$ for $5 \mathrm{~min}$ followed by 40 cycles of denaturation at $95^{\circ} \mathrm{C}$ for $30 \mathrm{sec}$, annealing at $60^{\circ} \mathrm{C}$ for $30 \mathrm{sec}$ and extension at $72^{\circ} \mathrm{C}$ for $45 \mathrm{sec}$. The sequences of the oligonucleotide primers used for this
Table I. Composition of the purified high-fat (D12337) and control (D12336) diets.

\begin{tabular}{|c|c|c|c|c|}
\hline \multirow[b]{3}{*}{ Ingredient } & \multicolumn{4}{|c|}{ Diet } \\
\hline & \multicolumn{2}{|c|}{ D12336 } & \multicolumn{2}{|c|}{ D12337 } \\
\hline & grams & kcal & grams & kcal \\
\hline Casein, 30 Mesh & 75 & 300 & 75 & 300 \\
\hline Soy protein & 130 & 520 & 130 & 520 \\
\hline DL-Methionine & 2 & 8 & 2 & 8 \\
\hline Corn starch & 275 & 1100 & 522.5 & 2090 \\
\hline Maltodextrin & 150 & 600 & 150 & 600 \\
\hline Sucrose & 30 & 120 & 30 & 120 \\
\hline Cellulose, BW200 & 90 & 0 & 90 & 0 \\
\hline Soybean oil & 50 & 450 & 50 & 450 \\
\hline Cocoa butter & 75 & 675 & 0 & 0 \\
\hline Coconut oil, 76 & 35 & 315 & 0 & 0 \\
\hline Mineral mix S10001 & 35 & 0 & 35 & 0 \\
\hline Calcium carbonate & 2.2 & 0 & 5.5 & 0 \\
\hline Sodium chloride & 8 & 0 & 8 & 0 \\
\hline Potassium citrate & 10 & 0 & 10 & 0 \\
\hline Vitamin mix V10001 & 10 & 40 & 10 & 40 \\
\hline Choline bitartrate & 2 & 0 & 2 & 0 \\
\hline Cholesterol, USP & 12.5 & 0 & 0.3 & 0 \\
\hline Sodium cholic acid & 5 & 0 & 0 & 0 \\
\hline FD\&C Red Dye \#40 & 0.1 & 0 & 0 & 0 \\
\hline FD\&C Blue Dye \#1 & 0 & 0 & 0.1 & 0 \\
\hline Total & 1000.1 & 4126 & 1120.4 & 4126 \\
\hline
\end{tabular}

study were 5'-TCC ACC TTT GAT GAC ATG GA-3' (sense) and 5'-TTG GCC AGC ACT CTG TAA TG-3' (antisense) for CYP7A1 (product size, $171 \mathrm{bp}$ ); and 5'-CCA GGG TTT GGA ATT ATT TC-3' (sense) and 5'-GAA GAT AAA CCC TAA GGC TC-3' (antisense) for 1A (product size, 297 bp). The relative expression levels of CYP7A1 in each sample (normalized to that of 1A) were determined using RQ software (Applied Biosystems). All qPCR experiments were repeated twice.

Histological staining and analysis of liver tissues. Liver tissues were recovered, frozen in liquid nitrogen and stored at $-80^{\circ} \mathrm{C}$ until use. Frozen liver tissues were cut with a cryomicrotome (CM3050S; Leica Biosystems, Nussloch, Germany) into 5-mm thick sections, stained with Oil Red O (Sigma-Aldrich, St. Louis, MO, USA) and counterstained with Harris hematoxylin (Sigma-Aldrich). Aortic tissues were embedded in paraffin prior to sectioning (5- $\mu \mathrm{m}$ thick). The sections were deparaffinized in xylene, hydrated in descending grades of ethanol and then stained with Harris hematoxylin and eosin (Sigma-Aldrich). The stained sections were viewed and photographed with a microscope (BX51; Olympus, Tokyo, Japan) equipped with a digital camera (DP71; Olympus). All images were captured at x200 magnification.

Data analysis. Data are presented as the mean \pm standard error of the mean (SEM) and were analyzed with a one-way 
Table II. Body weights and daily food intake.

\begin{tabular}{lccc}
\hline Group & Baseline body weight $(\mathrm{g})$ & Body weight 4 weeks later $(\mathrm{g})$ & Daily food intake $(\mathrm{g})$ \\
\hline Vehicle & $36.9 \pm 0.5$ & $41.5 \pm 1.8$ & $3.77 \pm 0.8$ \\
NC & $36.0 \pm 1.3$ & $42.3 \pm 0.4$ & $3.24 \pm 0.8$ \\
PC & $35.6 \pm 2.1$ & $43.5 \pm 0.3$ & $3.83 \pm 1.7$ \\
LE5 & $35.8 \pm 2.8$ & $42.3 \pm 0.3$ & $4.16 \pm 1.1$ \\
LE10 & $35.4 \pm 2.1$ & $42.1 \pm 0.6$ & $4.00 \pm 1.2$ \\
LE20 & $35.3 \pm 1.5$ & $41.7 \pm 0.4$ & $4.12 \pm 1.1$ \\
\hline
\end{tabular}

LE5, 5\% L. edodes; LE10, 10\% L. edodes; LE20, 20\% L. edodes.

Table III. Values of serum chemistry.

\begin{tabular}{lcccccc}
\hline & \multicolumn{5}{c}{ Serum level (mg/dl) } \\
\cline { 2 - 7 } Variable & Vehicle & NC & PC & LE5 & LE10 \\
\hline Total cholesterol & $151.5 \pm 17.7$ & $285.2 \pm 23.3^{\mathrm{a}}$ & $239.2 \pm 15.8^{\mathrm{b}}$ & $231.5 \pm 23.4^{\mathrm{b}}$ & $\begin{array}{c}182.7 \pm 18.0^{\mathrm{b}, \mathrm{c}} \\
\text { LE20 }\end{array}$ \\
HDL-cholesterol & $92.8 \pm 7.2$ & $92.1 \pm 16.4$ & $108.7 \pm 13.3$ & $82.8 \pm 19.7$ & $97.2 \pm 12.0$ & $88.5 \pm 15.6$ \\
LDL-cholesterol & $7.1 \pm 3.0$ & $53.8 \pm 1.5^{\mathrm{a}}$ & $45.6 \pm 2.0^{\mathrm{b}}$ & $42.6 \pm 7.4^{\mathrm{b}}$ & $37.8 \pm 3.6^{\mathrm{b}, \mathrm{c}}$ & $24.5 \pm 3.7^{\mathrm{b}, \mathrm{c}}$ \\
Triglyceride & $8.2 \pm 1.4$ & $33.5 \pm 9.4^{\mathrm{a}}$ & $23.2 \pm 5.0^{\mathrm{b}}$ & $13 \pm 3.0^{\mathrm{b}, \mathrm{c}}$ & $9.6 \pm 1.1^{\mathrm{b}, \mathrm{c}}$ & $7.3 \pm 0.7^{\mathrm{b}, \mathrm{c}}$ \\
\hline
\end{tabular}

${ }^{\mathrm{a}} \mathrm{P}<0.05$ vs. vehicle; ${ }^{\mathrm{b}} \mathrm{P}<0.05$ vs. negative control (NC); ${ }^{\mathrm{P}}<0.05$ vs. positive control (PC). LE5, 5\% L. edodes; LE10, 10\% L. edodes; LE20, $20 \%$ L. edodes; HDL, high-density lipoprotein; LDL, low-density lipoprotein.

analysis of variance (ANOVA) followed by Tukey's multiple comparison test. Statistical analyses were performed using Prism Graph Pad (version 4.0; GraphPad Software Inc., San Diego, CA, USA). P<0.05 was considered to indicate a statistically significant difference.

\section{Results}

Effects of L. edodes on BW and daily dietary intake. The differences in BW and daily food intake between mice fed the control diet and those given an HFD for 4 weeks were evaluated. The mice from all six groups had similar BW gains, as shown in Table II. The BWs of the mice in the vehicle, $\mathrm{NC}$ and $\mathrm{PC}$ groups were all the same at the end of experiment. Groups fed with L.edodes (5, 10 and 20\%) also had similar BW gains compared with those of the other groups at 9 weeks of age.

Serum levels of T-CHO, HDL, LDL and TG in the hypercholesterolemic mice. Dietary supplementation with L. edodes reduced the serum levels of T-CHO, HDL, LDL and TG, as shown in Table III. At 4 weeks, the mice fed the HFD showed a marked increase in $\mathrm{T}-\mathrm{CHO}$ levels (to 285.2 \pm 23.3 compared with $151.5 \pm 17.7 \mathrm{mg} / \mathrm{dl}$ in the vehicle group). Dietary supplementation with eritadenine $(10 \mathrm{mg} / \mathrm{kg})$ or L. edodes $(5,10$ and $20 \%$ ) attenuated the rise in serum T-CHO, LDL and TG levels caused by consumption of an HFD. In the groups fed with L.edodes (5, 10 and 20\%), the levels of T-CHO, LDL and TG were reduced in a dose-dependent manner compared with those in the NC group.

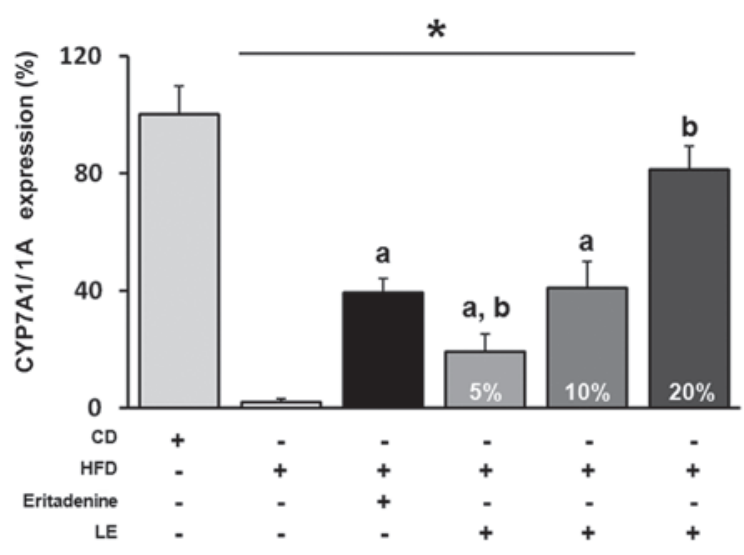

Figure 1. Effects of Lentinus (L.) edodes on cholesterol 7- $\alpha$-hydroxylase 1 (CYP7A1) mRNA expression in hypercholesterolemic mice. Quantitative polymerase chain reaction (qPCR) was performed to monitor the effects of L. edodes dietary supplementation on CYP7A1 mRNA expression in the liver of hypercholesterolemic mice. Liver tissues were from six groups of mice fed different diets: i) normal control diet (vehicle; CD); ii) high-fat diet (HFD) alone (negative control; NC); iii) HFD + eritadenine (positive control); iv) HFD + 5\% (w/w) L.edodes (LE5); v) HFD + 10\% L.edodes (LE10); and vi) HFD $+20 \%$ L. edodes (LE20). " $\mathrm{P}<0.05$ compared with the vehicle group; ${ }^{a} \mathrm{P}<0.05$ compared with the $\mathrm{NC}$; ${ }^{\mathrm{b}} \mathrm{P}<0.05$ compared with the $\mathrm{PC}$.

Expression of CYP7A1 mRNA in the liver of hypercholesterolemic mice. The expression of hepatic CYP7A1 was measured in the six groups of mice. As seen in Fig. 1, the level of CYP7A1 mRNA was reduced by HFD administration in the $\mathrm{NC}$ group compared with the level in the vehicle group and 
A
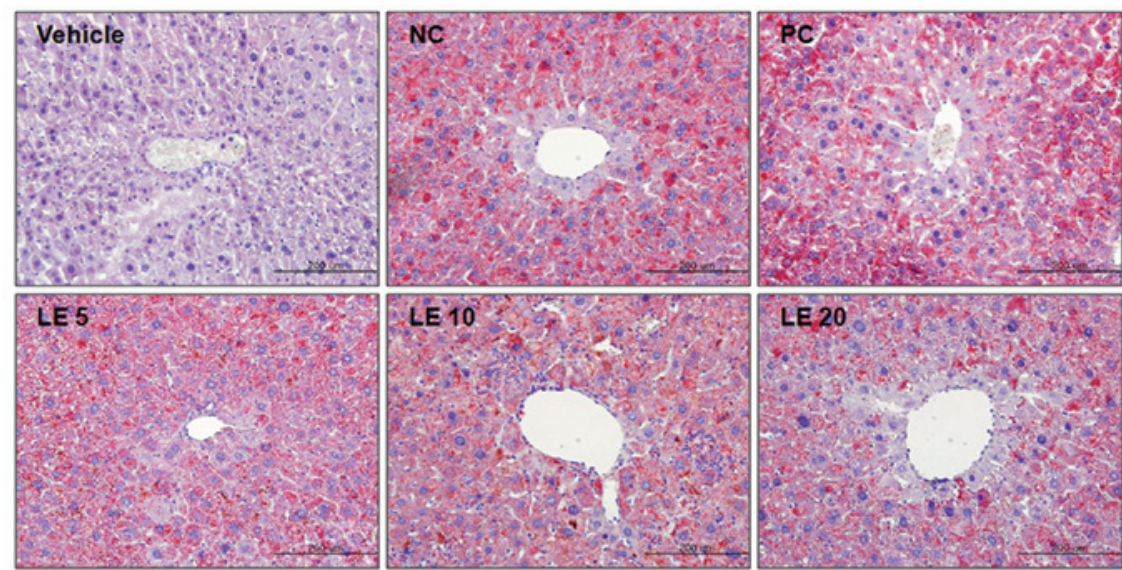

B

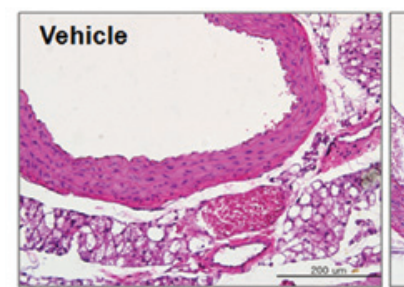

NC
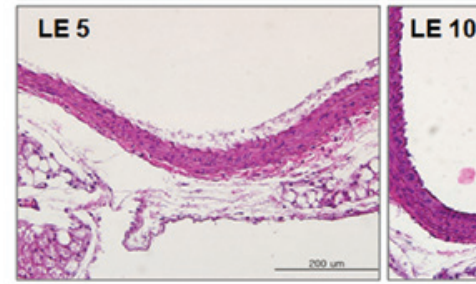

10
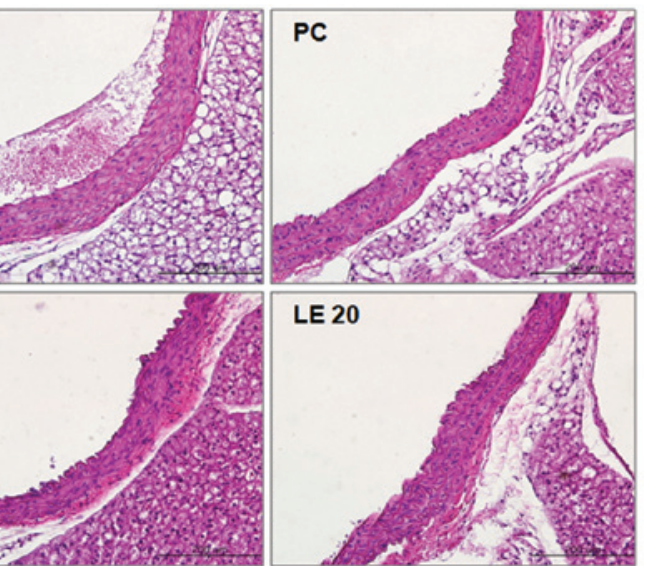

LE 20

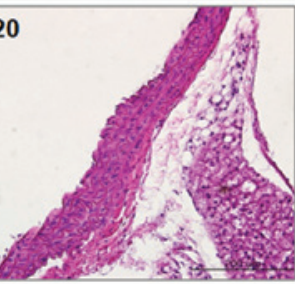

Figure 2. Effects of Lentinus (L.) edodes on hypercholesterolimic mouse liver and aorta histopathology. (A) Liver sections (stained with Oil Red O); (B) aorta sections (stained with hematoxylin and eosin). Vehicle, control diet alone; negative control (NC), high-fat diet (HFD) alone; positive control (PC), HFD + eritadenine; LE5, HFD + 5\% L. edodes; LE10, HFD + 10\% L. edodes; LE20, HFD + 20\% L. edodes. Magnification, x200.

was significantly increased by eritadenine supplementation in the PC group. Similar to the PC group, hepatic CYP7A1 mRNA expression was increased by $L$. edodes supplementation in a dose-dependent manner.

Effects of L. edodes on the histology of hypercholesterolemic mouse liver. The effect of L.edodes on liver tissue morphology in hypercholesterolemic mice is presented in Fig. 2A. Liver tissues were stained with Oil Red $\mathrm{O}$ and hematoxylin without eosin. The hepatic cords were arranged in a typical manner and located near the central vein in all groups of mice. Lipid droplets, evidence of fat accumulation in the cytoplasm of hepatocytes, were observed in the livers of hypercholesterolemic mice fed the HFD. The lipid accumulation was reduced by eritadenine or L. edodes supplementation.

Effects of L. edodes on the aortae of hypercholesterolemic mice. The effect of L.edodes on the descending aortic tissues of hypercholesterolemic mice is shown in Fig. 2B. Descending aortic tissues were stained with hematoxylin and eosin. Atherosclerotic plaques were observed in mice fed the HFD and the atherosclerotic plaques observed in the PC mice were smaller than those in the mice fed the HFD alone. The atherosclerotic plaques observed in the mice fed with the HFD and L. edodes supplementation were smaller and/or less numerous compared with the plaques observed in the NC (and/or PC) mice.

\section{Discussion}

L. edodes contains relatively high concentrations of vitamins D, B6, B9 and B12, as well as other beneficial compounds, such as eritadenine and dietary fiber (1). This mushroom has been proposed to be useful for treating hypercholesterolemia, inflammation, hypertension and osteoporosis (18-20). In a previous study, we examined the anti-osteoporotic effects of L. edodes and the ability of this fungus to induce the expression of duodenal and renal calcium transport channels in mice with osteoporosis-like symptoms (20). In the present study, we investigated the hypolipidemic effects of $L$. edodes and determined whether this fungus was able to prevent increases in T-CHO, LDL and TG levels in mice fed an HFD. Having fed the mice the experimental diets for 4 weeks, it was observed that dietary L. edodes supplementation reduced serum lipid levels, as well as fat accumulation, in the liver and descending aorta of the hypercholesterolemic mice. Eritadenine was demonstrated to have a similar effect. Previously, eritadenine was shown to lower phosphatidylcholine levels in rats $(3,21)$. An analysis of the eritadenine levels in L.edodes showed that the concentration of this compound ranged between 3.2 and $6.3 \mathrm{mg} / \mathrm{g}$ of dried mushrooms (22).

The stimulation of murine CYP7A1 gene expression by dietary cholesterol has been well studied (23-25). Oxysterols regulate the activity of responsive promoters in this gene by activating nuclear receptors known as LXRs (25). In addition, 
inactivation of the murine gene encoding the LXR $\alpha$ isoform has been shown to result in resistance to the stimulation of CYP7A1 gene expression by dietary cholesterol (23). CYP7A1 is the key regulatory enzyme of bile acid synthesis, plays a crucial role in cholesterol metabolism and has been implicated in genetic susceptibility to atherosclerosis (26). A CYP7A1 polymorphism has also been demonstrated to increase the progression of atherosclerosis in male patients (27). Furthermore, the development of fatty liver and hypercholesterolemia has been prevented by regulating the expression of CYP7A1 (28).

In the present study, atherosclerotic plaques were observed in the descending aortae of hypercholesterolemic mice. Plaque formation induced by the consumption of an HFD was effectively reduced by L.edodes supplementation (Fig. 2B). Hepatic fat accumulation was also observed in the livers of hypercholesterolemic mice. This was inhibited by eritadenine and L. edodes supplementation. Furthermore, CYP7A1 mRNA expression was decreased in the hypercholesterolemic mice and upregulated by eritadenine, as well as by L.edodes $(5$, 10 and $20 \%$ ), in a dose-dependent manner compared with the levels in the NC group.

In conclusion, the present study demonstrated that supplementation with eritadenine and L.edodes significantly inhibited the development of hypercholesterolemia induced by an HFD. The reductions in serum lipid levels, hepatic fat accumulation and aortic atherosclerotic plaque formation in mice treated with L. edodes illustrated the beneficial effects of this fungus. In addition, dietary supplementation with eritadenine or L.edodes increased the level of hepatic CYP7A1 expression in the hypercholesterolemic mice up to that observed in the vehicle control. Based on these results, we propose that the beneficial compounds present in L.edodes may be used in the treatment of hypercholesterolemia.

\section{Acknowledgements}

This study was supported by the National Research Foundation of Korea (NRF; grant no. 2010-0011433), funded by the Republic of Korea government (MEST).

\section{References}

1. Wasser SP: Shiitake (Lentinus edodes). In: Encyclopedia of Dietary Supplements DOI: 10.1081/E-EDS-120024880. Marcel Dekker, New York, pp. 653-664, 2005.

2. Ngai PH and Ng TB: Lentin, a novel and potent antifungal protein from shitake mushroom with inhibitory effects on activity of human immunodeficiency virus-1 reverse transcriptase and proliferation of leukemia cells. Life Sci 73: 3363-3374, 2003.

3. Shimada Y, Morita T and Sugiyama K: Eritadenine-induced alterations of plasma lipoprotein lipid concentrations and phosphatidylcholine molecular species profile in rats fed cholesterol-free and cholesterol-enriched diets. Biosci Biotechnol Biochem 67: 996-1006, 2003.

4. Sugiyama K, Akachi T and Yamakawa A: Hypocholesterolemic action of eritadenine is mediated by a modification of hepatic phospholipid metabolism in rats. J Nutr 125: 2134-2144, 1995.

5. Shimada Y, Morita T and Sugiyama K: Dietary eritadenine and ethanolamine depress fatty acid desaturase activities by increasing liver microsomal phosphatidylethanolamine in rats. J Nutr 133: 758-765, 2003.

6. Fukada S, Setoue M, Morita T and Sugiyama K: Dietary eritadenine suppresses guanidinoacetic acid-induced hyperhomocysteinemia in rats. J Nutr 136: 2797-2802, 2006.
7. Adachi R, Honma Y, Masuno H, et al: Selective activation of vitamin D receptor by lithocholic acid acetate, a bile acid derivative. J Lipid Res 46: 46-57, 2005.

8. Teo NH, Scott JM, Reed B, Neale G and Weir DG: Bile acid inhibition of vitamin B12 binding by intrinsic factor in vitro. Gut 22: 270-276, 1981

9. Seo YK, Chung YT, Kim S, Echchgadda I, Song CS and Chatterjee B: Xenobiotic- and vitamin D-responsive induction of the steroid/bile acid-sulfotransferase Sult2A1 in young and old mice: the role of a gene enhancer in the liver chromatin. Gene 386: 218-223, 2007.

10. Russell DW and Setchell KD: Bile acid biosynthesis. Biochemistry 31: 4737-4749, 1992.

11. Hubacek JA and Bobkova D: Role of cholesterol 7alpha-hydroxylase (CYP7A1) in nutrigenetics and pharmacogenetics of cholesterol lowering. Mol Diagn Ther 10: 93-100, 2006.

12. Moon MS, Lee MS, Kim CT and Kim Y: Dietary chitosan enhances hepatic CYP7A1 activity and reduces plasma and liver cholesterol concentrations in diet-induced hypercholesterolemia in rats. Nutr Res Pract 1: 175-179, 2007.

13. Chawla A, Saez E and Evans RM: 'Don't know much bile-ology'. Cell 103: 1-4, 2000.

14. Liu Y, Wu H, Wu W, Li R and Huang Y: Intervention of Huanglian Jiedu decoction on haemorheology and cholesterol-supplemented diet. Zhongguo Zhong Yao Za Zhi 34: 600-604, 2009 (In Chinese).

15. Tuncer MA, Yaymaci B, Sati L, et al: Influence of Tribulus terrestris extract on lipid profile and endothelial structure in developing atherosclerotic lesions in the aorta of rabbits on a high-cholesterol diet. Acta Histochem 111: 488-500, 2009.

16. von Holt K, Lebrun S, Stinn W, Conroy L, Wallerath T and Schleef R: Progression of atherosclerosis in the Apo E-/- model: 12-month exposure to cigarette mainstream smoke combined with high-cholesterol/fat diet. Atherosclerosis 205: 135-143, 2009.

17. Chibata I, Okumura K, Takeyama S and Kotera K: Lentinacin: a new hypocholesterolemic substance in Lentinus edodes. Experientia 25: 1237-1238, 1969.

18. Kabir Y, Yamaguchi M and Kimura S: Effect of shiitake (Lentinus edodes) and maitake (Grifola frondosa) mushrooms on blood pressure and plasma lipids of spontaneously hypertensive rats. J Nutr Sci Vitaminol (Tokyo) 33: 341-346, 1987.

19. Carbonero ER, Gracher AHP, Komura DL, et al: Lentinus edodes heterogalactan: Antinociceptive and anti-inflammatory effects. Food Chem 111: 531-537, 2008.

20. Lee GS, Byun HS, Yoon KH, Lee JS, Choi KC and Jeung EB: Dietary calcium and vitamin D2 supplementation with enhanced Lentinula edodes improves osteoporosis-like symptoms and induces duodenal and renal active calcium transport gene expression in mice. Eur J Nutr 48: 75-83, 2009.

21. Takashima K, Sato C, Sasaki Y, Morita T and Takeyama S: Effect of eritadenine on cholesterol metabolism in the rat. Biochem Pharmacol 23: 433-438, 1974.

22. Enman J, Rova U and Berglund KA: Quantification of the bioactive compound eritadenine in selected strains of shiitake mushroom (Lentinus edodes). J Agric Food Chem 55: 1177-1180, 2007.

23. Peet DJ, Turley SD, Ma W, et al: Cholesterol and bile acid metabolism are impaired in mice lacking the nuclear oxysterol receptor LXR alpha. Cell 93: 693-704, 1998.

24. Torchia EC, Cheema SK and Agellon LB: Coordinate regulation of bile acid biosynthetic and recovery pathways. Biochem Biophys Res Commun 225: 128-133, 1996.

25. Agellon LB, Drover VA, Cheema SK, Gbaguidi GF and Walsh A: Dietary cholesterol fails to stimulate the human cholesterol 7alpha-hydroxylase gene (CYP7A1) in transgenic mice. J Biol Chem 277: 20131-20134, 2002.

26. Lambrinoudaki I, Kaparos G, Papadimitriou D, et al: Methylenetetrahydrofolate reductase C677T polymorphism is associated with central adiposity and increased androgenicity in healthy postmenopausal women. Eur J Endocrinol 159: 233-241, 2008.

27. Hofman MK, Princen HM, Zwinderman AH and Jukema JW: Genetic variation in the rate-limiting enzyme in cholesterol catabolism (cholesterol 7alpha-hydroxylase) influences the progression of atherosclerosis and risk of new clinical events. Clin Sci (Lond) 108: 539-545, 2005.

28. Yiu WF, Kwan PL, Wong CY, et al: Attenuation of fatty liver and prevention of hypercholesterolemia by extract of Curcuma longa through regulating the expression of CYP7A1, LDL-receptor, HO-1, and HMG-CoA reductase. J Food Sci 76: H80-H89, 2011. 